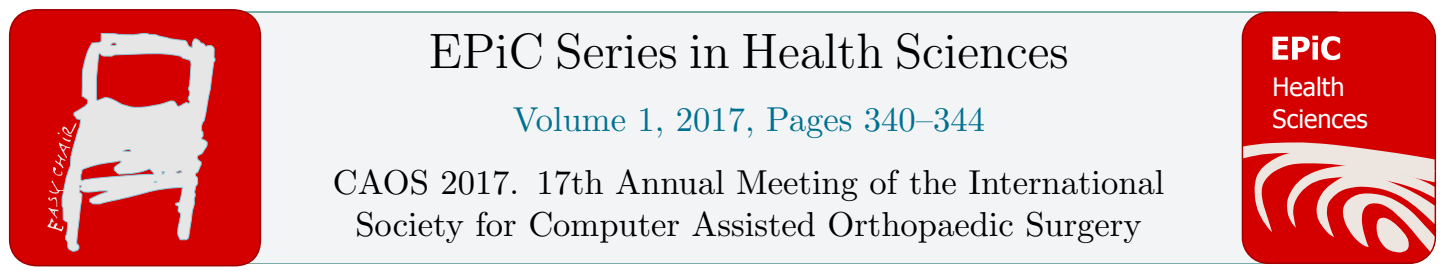

\title{
Hip Center Localization in Computer Assisted Orthopaedic Surgery: An In-Vitro Study
}

\author{
Zoheir Dib ${ }^{1,3}$, Guillaume Dardenne ${ }^{1,2}$, Chafiaa Hamitouche ${ }^{1,4}$, Christian \\ Lefèvre ${ }^{1,2,3}$ and Eric Stindel ${ }^{1,2,3}$ \\ ${ }^{1}$ LaTIM, INSERM, UMR 1101, SFR IBSAM, Brest, 29200, France. \\ ${ }^{2}$ CHU Brest, Brest, 29200, France. \\ ${ }^{3}$ Université de Bretagne Occidentale, Brest, 29200, France. \\ ${ }^{4}$ IMT-Atlantique, Dpt ITI, Brest, 29200, France. \\ guillaume.dardenne@chu-brest.fr
}

\begin{abstract}
The hip center (HC) in Computer Assisted Orthopedic Surgery (CAOS) can be determined either with anatomical (AA) or functional approaches (FA). AA is considered as the reference while FA compute the hip center of rotation (CoR). Four main FA can be used in CAOS: the Gammage, Halvorsen, pivot, and least-moving point (LMP) methods. The goal of this paper is to evaluate and compare with an in-vitro experiment (a) the four main FA for the HC determination, and (b) the impact on the HKA. The experiment has been performed on six cadavers. A CAOS software application has been developed for the acquisitions of (a) the hip rotation motion, (b) the anatomical HC, and (c) the HKA angle. Two studies have been defined allowing (a) the evaluation of the precision and the accuracy of the four FA with respect to the AA, and (b) the impact on the HKA angle. For the pivot, LMP, Gammage and Halvorsen methods respectively: (1) the maximum precision reach $14.2,22.8,111.4$ and 132.5 $\mathrm{mm}$; (2) the maximum accuracy reach 23.6, 40.7, 176.6 and $130.3 \mathrm{~mm}$; (3) the maximum error of the frontal HKA is $2.5^{\circ}, 3.7^{\circ}, 12.7^{\circ}$ and $13.3^{\circ}$; and (4) the maximum error of the sagittal HKA is $2.3^{\circ}, 4.3^{\circ}, 5.9^{\circ}, 6.1^{\circ}$. The pivot method is the most precise and accurate approach for the $\mathrm{HC}$ localization and the HKA computation.
\end{abstract}

\section{Introduction}

Two approaches can be used to compute the hip center (HC) in CAOS: anatomical and functional approaches. The anatomical approach is based on the morphology of the patient and corresponds to the center of a sphere fitted to a point cloud acquired with a digitizer on the surface of the femoral head. It requires however a direct access to the femoral head and is mainly used in hip replacement 
applications. Functional approaches are therefore often preferred in a context of Minimal Invasive Surgery. These approaches use the biomechanical properties of the hip and are based on the computation of the center of rotation (CoR) of the femur with respect to the pelvis. Several functional approaches have been described in the literature which are divided into two categories (Ehrig, 2006): the Sphere Fitting Methods (SFM) and the Coordinate Transformation Techniques (CTT).

SFM are based on the assumption that the recorded positions of the femoral marker fit perfectly a sphere. By considering that the femoral head is perfectly spherical, the $\mathrm{HC}$ is equivalent to the sphere center. The two main SFM methods in the literature are described by Gamage (SFG) (Gamage, 2002) and Halvorsen (SFH) (Halvorsen, 2003). CTT however considers the distance between markers on each joint segment, to enable the definition of a local coordinate system (Ehrig, 2006). The appropriate transformation of these local systems into a common reference system enables the approximation of a joint center. Two main methods are widely used: (a) the pivot (PIV) (Siston, 2006) which considers the CoR as a fixed point with respect to the femoral marker, and (b) the LeastMoving-Point (LMP) method (Marin, 2003) (Stindel, 2005), which consists of identifying the point that moves the least during the rotation motion.

The goal of this paper is to evaluate and compare with an in-vitro experiment (a) the four main methods, SFG, SFH, PIV and LMP for the computation of the HC, and (b) the impact on the HKA angle.

\section{Materials and Methods}

The experiment has been performed on six full cadaveric lower limbs. A femoral and a tibia markers were respectively attached to the femur and the tibia. A specific software application has been developed and was installed on a CAOS station. This software application was divided into four steps: (1) Acquisition of the circumduction motion by recording during the motion 500 transforms associated to the femoral marker, (2) acquisition of the knee center (KC) corresponding to the middle of the medial (MC) and lateral (LC) condyles of the knee, (3) acquisition of the ankle center corresponding to the middle of the medial and lateral malleolus, and (4) acquisition of the anatomical $\mathrm{HC}, H C_{\text {anat }}$, after the hip dislocation, corresponding to the center of a sphere fitted to 1,000 points acquired on the femoral head. All these steps have been performed five times per lower limbs.

A first study has been realized allowing the evaluation of (1) the precision and (2) the accuracy of the HC methods. The precision is defined as the distance between the mean HCs: $H C_{\text {mean_anat }}, H C_{\text {mean_LMP }}, H C_{\text {mean_PIV }}, H C_{\text {mean_SFG }}$ and $H C_{\text {mean_SFH }}$, and their respective $H C_{\text {anat }}, H C_{L M P}, H C_{P I V}, H C_{S F G}$ and $H C_{S F H}$ obtained from the five acquisitions. The accuracy is defined as the distance between the ground truth, $H C_{\text {mean_anat }}$ and the four HCs: $H C_{S F G}, H C_{S F H}, H C_{P I V}, H C_{L M P}$ obtained from the five acquisitions.

A second study has been realized allowing the assessment of the HKA angle. Four HKA angles, $H K A_{S F G}, H K A_{S F H}, H K A_{P I V}, H K A_{L M P}$, have been computed, corresponding to the angle between $\mathrm{KC}, \mathrm{AC}$, and respectively the four $\mathrm{HC}$ definitions: $H C_{S F G}, H C_{S F H}, H C_{P I V}, H C_{L M P}$. The $H K A_{\text {GroundTruth }}$ is defined as the HKA angle between $\mathrm{KC}, \mathrm{AC}$ and the ground truth $H C_{\text {mean_anat }}$. The error is defined as the difference between the $H K A_{\text {GroundTruth }}$ and the four HKA: $H K A_{S F G}, H K A_{S F H}, H K A_{P I V}$ and $H K A_{L M P}$. These errors have been projected onto the frontal and the sagittal planes. 


\section{Results}

Figure 1 shows the precision of $H C_{\text {anat }}$ (a), the precisions of $H C_{L M P}, H C_{P I V}, H C_{S F G}$ and $H C_{S F H}$ (b), and the accuracy of $H C_{L M P}, H C_{P I V}, H C_{S F G}$ and $H C_{S F H}$ (c).

(a)
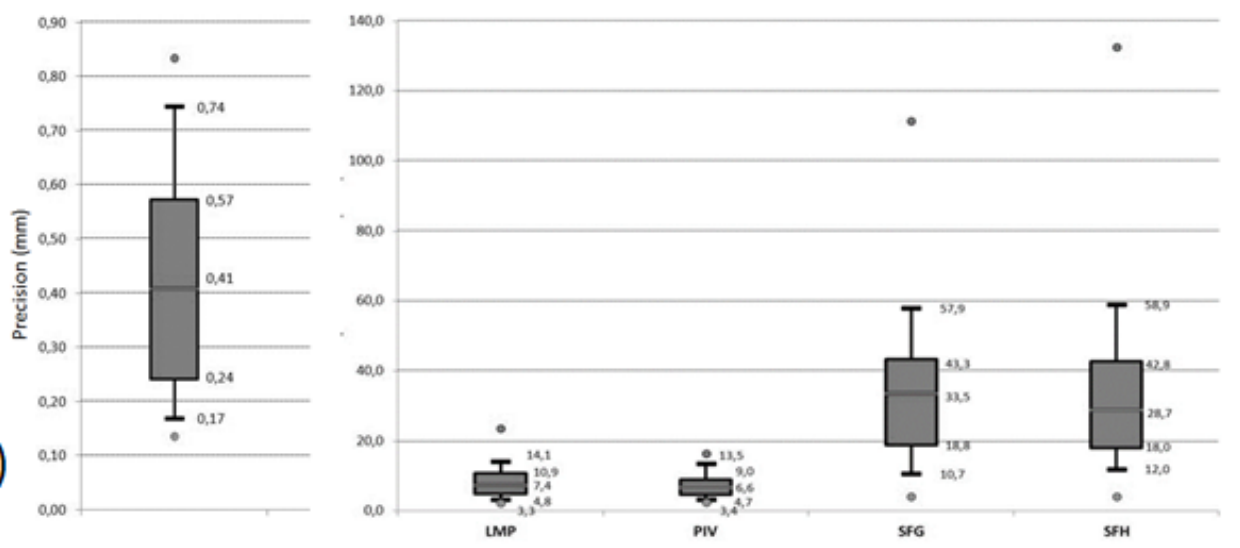

(b)

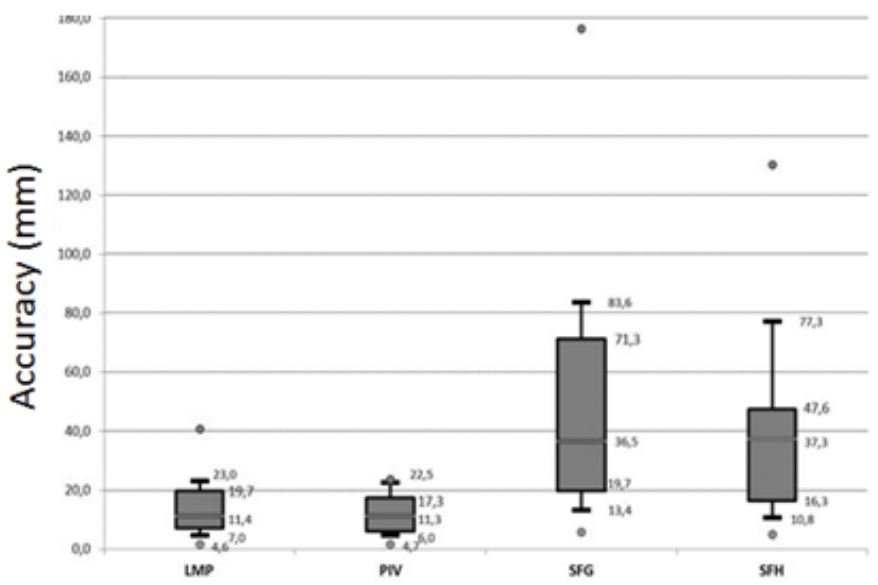

(c)

Figure 1: Precision of $H C_{\text {anat }}$ (a), $H C_{L M P}, H C_{P I V}, H C_{S F G}$ and $H C_{S F H}$ (b), and accuracy of $H C_{L M P}$, $H C_{P I V}, H C_{S F G}$ and $H C_{S F H}$.

Figure 2 shows the errors of $H K A_{L M P}, H K A_{P I V}, H K A_{S F G}$ and $H K A_{S F H}$ in the frontal plane (a), and in the sagittal plane (b). 


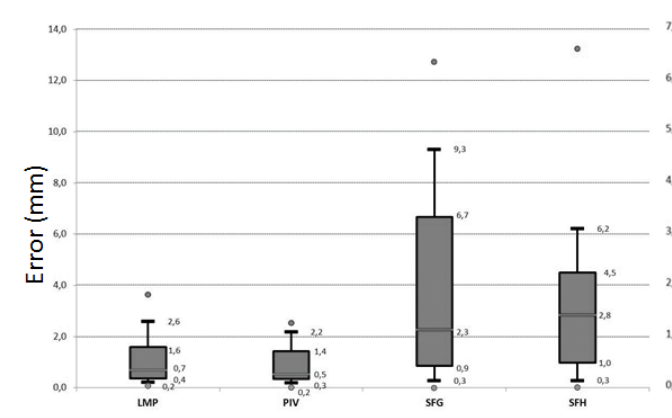

(a)

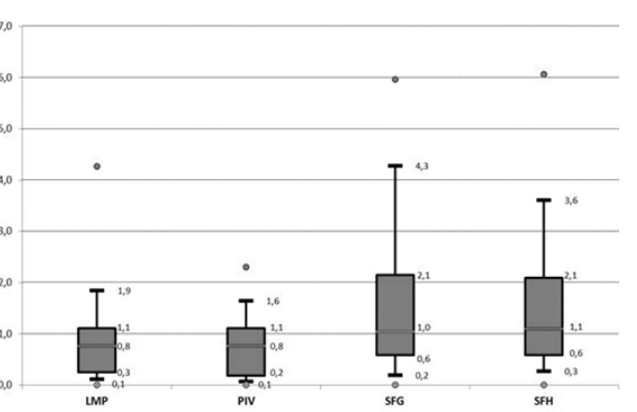

(b)

Figure 2: Errors of $H K A_{L M P}, H K A_{P I V}, H K A_{S F G}$ and $H K A_{S F H}$ in the frontal (a) and sagittal (b) planes

In the figures, the middle line represents the median, the central rectangle spans the first quartile to the third quartile and the vertical line extends from the first decile to the ninth decile. Minimum and maximum values are displayed as separated points.

\section{Discussion}

The maximal precision of $H C_{\text {anat }}$ is $0.83 \mathrm{~mm}$ and can be therefore considered as a reference for the assessment of the HC methods (Ehrig, 2006) (Dib, 2013).

CTT are more precise and accurate than SFM for (a) the HC determination and (b) the computation of the HKA angle.

In many clinical applications, the required accuracy for the determination of the HKA must be superior to $3^{\circ}$ (Bargren, 1983) (Ritter, 1994). SFM are therefore not adapted. The accuracy of the LMP approach is superior to SFM but a maximal error of $3.7^{\circ}$ has been recorded.

This in-vitro experiment show that the PIV method is the most precise and accurate approach for the determination of the functional $\mathrm{HC}$ and the computation of the HKA.

\section{References}

Bargren, J., Blaha, J., \& Freeman, M. (1983). Alignment in total knee arthroplasty: correlated biomechanical and clinical investigations. Clin Orthop, 173, 178-183.

Dib, Z. (2013). Detection of the hip center in computer-assisted surgery: An in vitro assessement study. IRBM, 34, 319-321.

Ehrig, R. (2006). A survey of formal methods for determining the centre of rotation of ball joints. Journal of Biomechanics, 39(15), 2798-2809.

Gamage, S. (2002). New least squares solutions for estimating the average center of rotation and the axis rotation. Journal of Biomechanics, 87-93.

Halvorsen, K. (2003). Bias compensated least squares estimate of the center of rotation. Journal of Biomechanics, 999-1008.

Marin, F. (2003). Accurate determination of a joint centre center based on the minimal amplitude point method. Computer Aided Surgery, 8, 30-34. 
Ritter, M., Faris, P., Keating, E., \& Meding, J. (1994). Postoperative alignment of total knee replacement: Its effect on survival. Clinical Orthopaedics and Related Research, 299, 153156.

Siston, R. (2006). Evaluation of a new algorithm to determine the hip joint center. Journal of Biomechanics, 39(1), 125-30.

Stindel, E. (2005). Detection of the center of the hip joint in computer-assisted surgery: an evaluation study of the Surgetics algorithm. Computer Aided Surgery, 10(3), 133-9. 